As expected, $\mathrm{NO}_{3}^{-}$supplementation led to a significant increase in plasma nitrite $(>8.5$-fold) and particularly nitrate ( $>28$-fold). Similar to our COPD trial, ${ }^{5}$ we observed marked variation in response to exogenous nitrate despite an identical dose and time interval (Figure $2 \mathrm{~b}$ and $\mathrm{c}$ ). We cannot explain these variations, but it is likely that differences in oral bacteria and stomach acidity, age and medication are contributing factors. Further, we recently reviewed data demonstrating a variation in metabolism of exogenously administered nitrate when measured at a single time-point (e.g., 3 hours), yet the extent of nitrite/nitrate production (across 24 hours) was found to be largely similar. ${ }^{7}$ The importance of our placebo-controlled design is illustrated by our earlier demonstration of intra-individual variability with repeat doses of $\mathrm{NO}_{3}^{-}$.

Pharmacologic preparations of organic nitrate are used, with varied success, to treat HF and NIDCM. It should be noted that, despite their similar physiologic effects, organic and inorganic nitrate (such as dietary nitrate) possess different chemical structures and pharmacokinetics. The potency of inorganic nitrate is much lower than that of organic nitrate. However, organic nitrate may result in tolerance and, when discontinued, rebound effects are often evoked. In contrast, inorganic, dietary nitrates do not show any signs of tolerance, and physiologic effects may be potentiated with long-term ingestion.

Although our trial has some major limitations, including the acute nature of the assessments and the small sample size, we utilized a robust trial design among a cohort of well-characterized NIDCM subjects. Dietary nitrate has

\section{Thrombus around the redesigned HeartWare HVAD inflow cannula: A pathologic case series}

Kyle C. Strickland, MD, PhD,

Jaclyn C. Watkins, MD, MS, ${ }^{a}$

Gregory S. Couper, MD, ${ }^{\mathrm{b}}$

Michael M. Givertz, MD, ${ }^{c}$ and

Robert F. Padera, MD, PhD ${ }^{a}$

From the ${ }^{a}$ Department of Pathology; ${ }^{b}$ Division of Cardiac Surgery; and the ${ }^{C}$ Cardiovascular

Division, Brigham and Women's Hospital,

Harvard Medical School, Boston,

Massachusetts

The HeartWare HVAD (HeartWare, Inc., Framingham, MA) is a centrifugal, continuous-flow left ventricular assist device (LVAD) that was approved by the US Food and Drug Administration in 2012 for use as a bridge to cardiac transplantation in patients at risk of death from refractory end-stage left ventricular heart failure. ${ }^{1}$ In contrast to other LVADs currently approved by the Food and Drug Administration, the pump itself resides within the thorax on the epicardial surface rather than in an abdominal or extracorporeal location. Early experience with the HVAD potential as a novel, therapeutic strategy to increase exercise tolerance in NIDCM. Our preliminary results require confirmation among larger samples in the long-term setting.

\section{Disclosure statement}

The authors have no conflicts of interest to disclose.

This study was financially supported by the Irish Heart Foundation (to C.K.) and, in part, by the HeartBeat Trust.

\section{References}

1. O'Neill JO, Young JB, Pothier CE, et al. Peak oxygen consumption as a predictor of death in patients with heart failure receiving beta-blockers. Circulation 2005;111:2313-8.

2. Zamani P, Rawat D, Shiva-Kumar P, et al. Effect of inorganic nitrate on exercise capacity in heart failure with preserved ejection fraction. Circulation 2015;131:371-80.

3. Coggan AR, Leibowitz JL, Spearie CA, et al. Acute dietary nitrate intake improves muscle contractile function in patients with heart failure: a double-blind, placebo-controlled, randomized trial. Circ Heart Fail Circ Heart Failure 2015;8:914-20.

4. Kenjale AA, Ham KL, Stabler T, et al. Dietary nitrate supplementation enhances exercise performance in peripheral arterial disease. J Appl Physiol (1985) 2011;110:1582-91.

5. Kerley CP, Cahill K, Bolger K, et al. Dietary nitrate supplementation in COPD: an acute, double-blind, randomized, placebo-controlled, crossover trial. Nitric Oxide 2015;44:105-11.

6. Gilchrist M, Winyard PG, Fulford J, et al. Dietary nitrate supplementation improves reaction time in type 2 diabetes: development and application of a novel nitrate-depleted beetroot juice placebo. Nitric Oxide 2014;40:67-74.

7. James PE, Willis GR, Allen JD, et al. Nitrate pharmacokinetics: taking note of the difference. Nitric Oxide 2015;48:44-50.

demonstrated acceptable survival to transplant but raised concerns about the risk of stroke, ${ }^{2}$ presumably from thromboembolic events. In the ADVANCE study, the HVAD was shown to be non-inferior to contemporaneously implanted commercially available LVADs in a bridge-totransplant trial $^{3}$ and in continued access protocols. ${ }^{4}$ Recent "real world" outcomes have also been favorable, including analysis of a post-market device registry in the European Union and Australia. ${ }^{5}$ Thrombosis is an adverse event that can be seen with any mechanical circulatory support device, and the HVAD is no exception. ${ }^{6}$ In May 2011, a design change was implemented for the outer surface of the HVAD inflow cannula from a completely smooth, polished titanium surface to one that has a collar of sintered titanium microspheres that cover the half of the cannula adjacent to the pump, ${ }^{7}$ with the goal of preventing tissue growth beyond the sintered area of the cannula.

There have been no formal pathologic studies of HVADs that have been removed at the time of pump exchange, successful bridge to transplant, or autopsy. We report here a case series of 8 consecutive HVADs with the current sintered inflow cannula in patients who were successfully bridged to transplant at Brigham and Women's Hospital from December 30, 2013, to August 7, 2015. 
Table

Clinical and Pathologic Characteristics of Patients With HVADs and Explanted HeartsTable 1

\begin{tabular}{|c|c|c|c|c|c|c|c|c|c|}
\hline \multirow[b]{2}{*}{ Characteristic } & \multirow[b]{2}{*}{ Average } & \multicolumn{8}{|c|}{ Patient } \\
\hline & & 1 & 2 & 3 & 4 & 5 & 6 & 7 & 8 \\
\hline Sex & 7:1 (M:F) & $\mathrm{F}$ & M & M & M & M & M & M & M \\
\hline Age at implantation (years) & 47 & 23 & 60 & 32 & 56 & 38 & 41 & 67 & 61 \\
\hline Duration of implantation (days) & 186 & 195 & 228 & 156 & 118 & 71 & 116 & 460 & 145 \\
\hline Post-transplant interval (days) & 274 & 637 & 347 & 318 & 317 & 246 & 211 & 64 & 50 \\
\hline Diagnosis & & $\mathrm{DCM}^{\mathrm{a}}$ & DCM & IHD & DCM & DCM & IHD & DCM & DCM \\
\hline TTR $(\%)$ & 70 & NA & 46 & 64 & 81 & 72 & 78 & 82 & 69 \\
\hline LVIDd $(\mathrm{cm})$ & & 7.2 & 9.1 & 7.2 & 6.6 & 7.6 & 3.6 & 5.9 & 6.6 \\
\hline LVIDs (cm) & & 6.4 & 8.4 & 6.3 & 6.0 & 7.2 & 3.2 & 5.8 & 6.3 \\
\hline IVS thickness (cm) & & 0.4 & 0.8 & 0.7 & 1.1 & 0.9 & 1.4 & 0.9 & 1.0 \\
\hline LVPW thickness (cm) & & 0.6 & 0.8 & 1.3 & 1.0 & 0.9 & 1.4 & 0.8 & 1.1 \\
\hline Speed (rpm) & & NA & 2,700 & 2,700 & 2,720 & 2,680 & 2,480 & 2,680 & 2,600 \\
\hline Average flow (liter/min) & & NA & 5.0 & 6.2 & 4.1 & 5.6 & 4.7 & 4.8 & 4.7 \\
\hline Average LDH (U/liter) & & NA & 199 & 217 & 271 & 427 & 285 & 264 & 209 \\
\hline Explanted heart weight (g) & 656 & 270 & 920 & 753 & 762 & 560 & 575 & 624 & 789 \\
\hline Thrombus size $(\mathrm{cm})$ & 2.6 & 0.7 & 3.0 & 1.1 & 4.5 & 4.2 & 2.3 & 0.4 & 4.5 \\
\hline Contact of cannula with myocardium & $50 \%$ & Yes & No & Yes & No & No & Yes & No & Yes \\
\hline Pannus formation & $100 \%$ & Yes & Yes & Yes & Yes & Yes & Yes & Yes & Yes \\
\hline $\begin{array}{l}\text { Significant extension beyond } \\
\text { sintered aspect }\end{array}$ & $63 \%$ & No & Yes & Minimal & Yes & Minimal & Yes & Yes & Yes \\
\hline Embolic events & $38 \%$ & None & Splenic & Coronary $^{\mathrm{b}}$ & None & None & None & Stroke & None \\
\hline Other thrombus & $13 \%$ & None & $\begin{array}{l}\text { Outflow } \\
\text { graft }\end{array}$ & None & None & None & None & None & None \\
\hline Aortic cusp fusion & $13 \%$ & No & No & No & No & No & Yes & No & No \\
\hline
\end{tabular}

The institutional review board approved this study. All 8 patients were found to have thrombus associated with the outer aspect of the HVAD inflow cannula. Although our center has many patients currently with HVADs in place, these 8 patients represent all patients who have been transplanted to date after HVAD use.

The clinical and pathologic data are shown in the Table. The cohort included 7 men and 1 woman with a mean age of 47 years. The diagnoses included dilated cardiomyopathy in 5 patients, ischemic heart disease in 2 patients, and hypertrophic cardiomyopathy in 1 patient. In the absence of active bleeding or other contraindications, all patients received post-implantation anti-coagulation including fulldose aspirin and a continuous infusion of heparin within 2 to 3 days after implantation as a bridge to therapeutic warfarin sodium with a goal of an international normalized ratio of 2 to 3 . Anti-coagulation was monitored by a dedicated anticoagulation service; the average time in therapeutic range was 70\%. During HVAD implantation, the external centering site on the left ventricular apex was determined by inserting a transapical needle aiming at the mitral valve orifice. Postoperatively, chest x-rays, computed tomography scans, and echocardiography studies were reviewed to assess the position of the cannula. The only inflow cannula that showed sub-optimal positioning was from Patient 6 (recent acute myocardial infarction with a non-dilated left ventricle bridged to HVAD by extracorporeal membrane oxygenation), which had a horizontal lie aiming at the posterior wall. For the other patients, chest $\mathrm{x}$-ray and computed tomography demonstrated a desirable position of the inflow cannula. No patients demonstrated inflow cannula obstruction on any echocardiography studies during their implantation time.

The average time from ventricular assist device implantation to transplant was 186 days (range 71-460 days). During the period of support, complications included driveline and ventricular assist device-associated infections in 2 patients; there were no incidences of gastrointestinal bleeding. Clinical thromboembolic events occurred in 3 patients (stroke, splenic infarct, intracoronary thromboemboli); we did not screen radiologically for sub-clinical thromboembolic events. One patient experienced status epilepticus shortly after HVAD implantation, which was presumably due to an air embolus from the pump. All patients underwent successful transplantation and remain alive in the current post-transplant interval (mean 274 days, range 50-637 days).

Gross pathologic examination of all devices (8 of 8; $100 \%$ ) revealed thrombi on the outer aspect of the inflow cannula of the HVAD (Figure 1). The size of the thrombi ranged from 0.4 to $4.5 \mathrm{~cm}$ (average $2.6 \mathrm{~cm}$ ), with the largest 

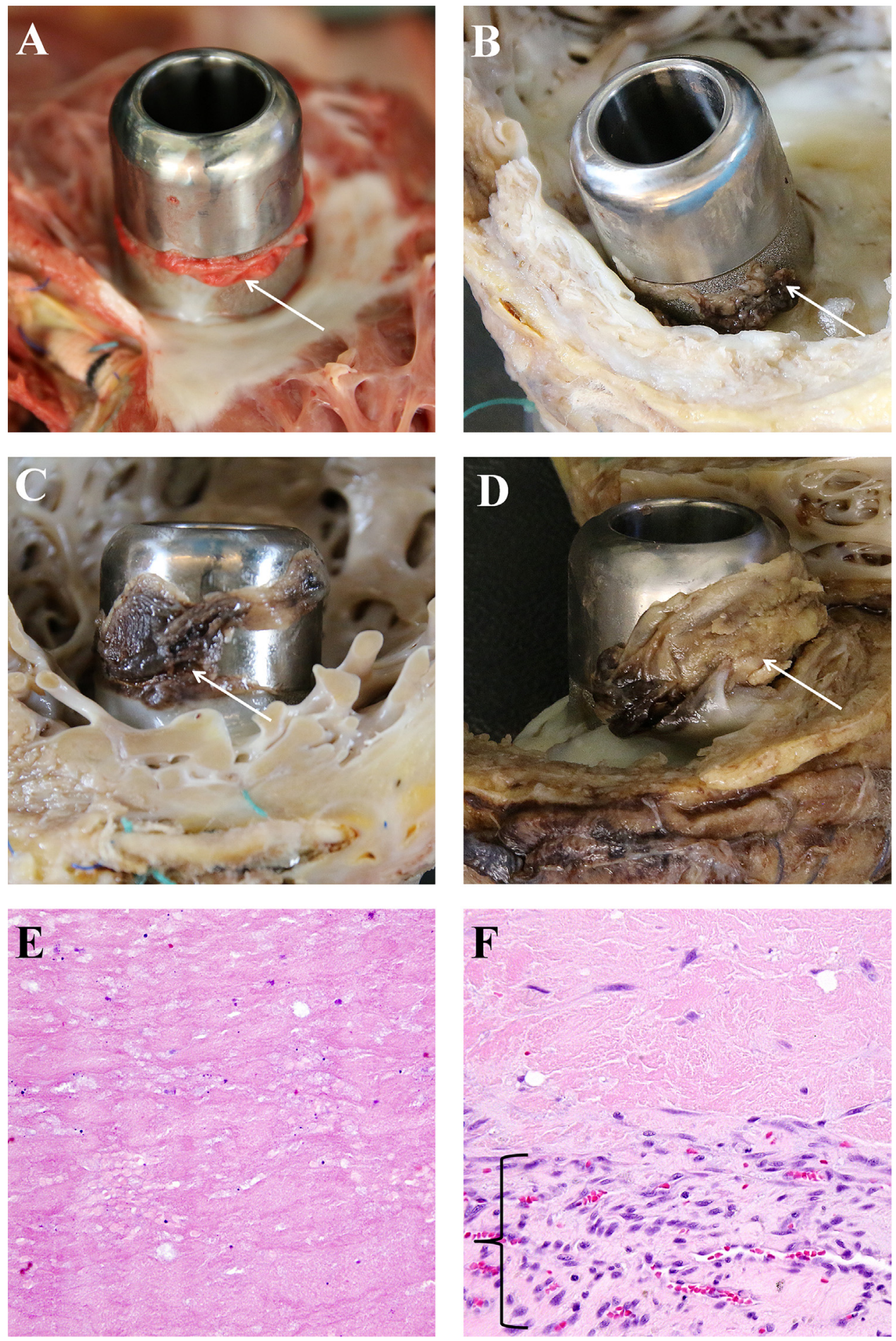

Figure 1 Thrombi associated with the outer surface of HVAD inflow cannulas. (A) Thrombus (arrow) at the junction between the sintered and smooth portion of the inflow cannula in a fresh specimen. (B) Thrombus (arrow) on the sintered portion of the cannula that does not extend onto the smooth portion of the cannula. (C) Thrombus (arrow) extending onto the smooth portion of the cannula. (D) Large thrombus (arrow) extending from the sintered portion onto the smooth portion of the cannula. (E) Histologic image of bland, laminated unorganized thrombus (hematoxylin-eosin stain, magnification $\times 400)$. (F) Histologic image of thrombus with area of organization (bracket) (hematoxylin-eosin stain, magnification $\times 400$ ).

present circumferentially around the outer aspect of the inflow cannula. The thrombus in 1 case formed at the junction of the sintered and non-sintered portions of the cannula (Figure 1A) with minimal extension onto the non-sintered portion. Thrombi in 2 cases either were confined to the sintered portion of the cannula (Figure 1B) or had minimal extension onto the non-sintered portion. In 5 devices, the thrombus extended substantially beyond the sintered portion of the inflow cannula to involve the smooth portion (Figure 1C and D). Histologic evaluation of these thrombi revealed bland, 
laminated thrombus (Figure 1E) with no evidence of infection. In some cases, there was evidence of organization in the areas of thrombus adjacent to the apical myocardium (Figure 1F). No thrombus was apparent inside any of the pumps. In comparison, 16 patients at our institution were bridged to transplant with the Thoratec (Pleasanton, CA) HeartMate II LVAD over the same time period; only 1 of the explanted pathologic specimens showed thrombosis around the inflow cannula (1 of $16,6.25 \%$ ).

All (8 of $8 ; 100 \%)$ of the HVADs showed fibrous pannus overgrowth over a portion of the inflow cannula starting from its junction with the left ventricular wall; none of the pannus reached above the sintered portion of the inflow cannula. There was evidence of contact of the inflow cannula with the left ventricular myocardium in 4 of 8 cases, without evidence of significant obstruction to flow, abrasion, or thrombus formation in the contacting areas on the endomyocardial surface. The gross examinations of the other portions of the HVADs were unremarkable, with no evidence of epicardial damage, driveline infection, or driveline damage.

There seems to be some confusion in the HVAD literature regarding the term pannus as it relates to material found on the inflow cannula. Najjar et $\mathrm{al}^{6}$ reported the formation of an "eccentric pannus" in association with HVAD inflow cannulas; however, the gross appearance and morphology indicates that this was actually thrombus, consistent with the findings reported herein. Additionally, what was labeled as "healed scar" was endocardial fibrosis with a thin layer of pannus formation over the sintered portion of the inflow cannula.

The 3 elements of Virchow's triad are the blood contacting surface, the nature of blood flow over said surface, and the intrinsic coagulability of blood in a given patient. When these factors are perturbed, thrombi are more likely to form. In the case of the HVAD inflow cannula, the metallic surface provides a nidus for thrombus formation. Despite the redesign of the HVAD inflow cannula, we observed thrombus on all the devices in our cohort, and significant thrombus extended from the sintered area onto the smooth area of the cannula in $62.5 \%$ of our cases. There was minimal to mild pannus formation on the cannulas, none of which extended beyond the sintered portion.

Potential factors that may predispose to thrombosis around the HVAD inflow cannula include the following: (1) an efficient flow path through the mitral valve and into the inflow cannula may lead to stasis or slowed flow in the "gutter" between the tip of the inflow cannula and the apical left ventricular myocardium, especially in hearts with poor apical contractility and at higher pump speeds; (2) changes to the shape and compliance of the left ventricular apex as a result of the epicardial pump placement may impact the above-described stasis issue; (3) the ring of discontinuity between the sintered and non-sintered portion of the pump may precipitate flow disturbances, where the thrombus was centered in 1 of our cases; (4) close proximity of the pump to the inflow cannula may be associated with an increase in the temperature of the inflow cannula as a result of heat generation from the pump that may not be adequately dissipated, potentially exacerbated at higher mean arterial pressures; and (5) similarly, vibration or other mechanical forces may be more easily transferred to the intracavitary portion of the inflow cannula because of the proximity of the pump and inflow cannula. These device-specific factors would be superimposed on others known to predispose to LVAD thrombosis, including patient factors (e.g., intrinsic hypercoagulability, hypertension), management factors (e.g., insufficient anticoagulation), and inflow cannula position. We believe that it is likely a combination of some of these factors that gives rise to thrombus formation.

This is a single-center, real-world, retrospective, uncontrolled observational study, with 7 of the 8 patients transplanted within 8 months of HVAD implantation. Although the number of devices is small, this study represents the largest pathologic series in the literature to date. These are important limitations that hamper the ability to determine the true incidence of thrombosis on the outer surface of the redesigned HVAD inflow cannula as well as the underlying causes or any clinical implications, if present. Nevertheless, this brief report should encourage other groups to examine these devices carefully at the time of exchange, explantation, or autopsy and to share their findings in the literature.

In conclusion, we report the presence of thrombus around the inflow cannula in $100 \%$ of patients with HVADs as bridge to transplant in our case series. It is possible that these thrombi represent the source of some of the embolic events observed with this device.

\section{Disclosure statement}

None of the authors has a financial relationship with a commercial entity that has an interest in the subject of the presented manuscript or other conflicts of interest to disclose.

The authors thank David Deicicchi, PharmD of the Brigham and Women's Hospital Anticoagulation Service for the time in therapeutic range calculations and Dr. Frederick Schoen and Dr. Richard Mitchell of the Brigham and Women's Hospital Department of Pathology for their thoughtful review of this manuscript.

\section{References}

1. Larose JA, Tamez D, Ashenuga M, Reyes C. Design concepts and principle of operation of the HeartWare ventricular assist system. ASAIO J 2010;56:285-9.

2. Bashir J, Legare J-F, Freed DH, Cheung A, Rao V, Toma M. Multicentre Canadian experience with HeartWare ventricular assist device: concerns about adverse neurological outcomes. Can J Cardiol 2014;30:1662-7.

3. Aaronson KD, Slaughter MS, Miller LW, et al. Use of an intrapericardial, continuous-flow, centrifugal pump in patients awaiting heart transplantation. Circulation 2012;125:3191-200.

4. Slaughter MS, Pagani FD, McGee EC, et al. HeartWare ventricular assist system for bridge to transplant: combined results of the bridge to transplant and continued access protocol trial. J Heart Lung Transplant 2013;32:675-83. 
5. Strueber M, Larbalestier R, Jansz P, et al. Results of the postmarket Registry to Evaluate the HeartWare Left Ventricular Assist System (ReVOLVE). J Heart Lung Transplant 2014;33:486-91.

6. Najjar SS, Slaughter MS, Pagani FD, et al. An analysis of pump thrombus events in patients in the HeartWare ADVANCE bridge to transplant and continued access protocol trial. J Heart Lung Transplant 2014;33:23-34.

7. Soltani S, Kaufmann F, Vierecke J, et al. Design changes in continuous-flow left ventricular assist devices and life-threatening pump malfunction. Eur J Cardiothorac Surg 2015;47:984-9.

\section{Evaluation of the effect of diminished pulsatility as seen in continuous flow ventricular assist devices on arterial endothelial cell phenotype and function}

\author{
Phani K. Patibandla, PhD, ${ }^{a, 1}$ \\ Namakkal S. Rajasekaran, PhD, , ,e,1,2 \\ Sandeep B. Shelar, PhD, ${ }^{\text {b }}$ \\ Guruprasad A. Giridharan, PhD, \\ Silvio H. Litovsky, MD, ${ }^{d}$ and \\ Palaniappan Sethu, PhD ${ }^{a, f, 2}$ \\ From the ${ }^{a}$ Division of Cardiovascular Disease, \\ Department of Medicine \\ and ${ }^{b}$ Division of Molecular and Cellular \\ Pathology, Department of Pathology, \\ University of Alabama at Birmingham, \\ Birmingham, Alabama; ${ }^{c}$ Department of \\ Bioengineering, Speed School of Engineering, \\ University of Louisville, Louisville, Kentucky; \\ ${ }^{d}$ Department of Pathology, University of \\ Alabama at Birmingham, Birmingham, \\ Alabama; ${ }^{e}$ Division of Cardiovascular \\ Medicine, Department of Medicine, University \\ of Utah School of Medicine, Salt Lake City, \\ Utah; and the ${ }^{f}$ Department of Biomedical \\ Engineering, School of Engineering, University \\ of Alabama at Birmingham, Birmingham, \\ Alabama
}

Continuous-flow ventricular assist devices (CFVADs) have emerged as a standard therapy for patients with advanced heart failure because of the paucity of donor organs. CFVADs are preferred over pulsatile-flow ventricular assist devices (PFVADs) due to inherent technologic and clinical advantages of CFVADs, including smaller size, higher durability and energy efficiency, lower pump thrombosis rates, and reduced surgical trauma during device implantation. ${ }^{1}$ Although CFVADs and PFVADs both unload the native ventricle and augment cardiac output, full CFVAD support significantly diminishes arterial pulsatility, as quantified by pulse pressure $(>75 \%$ reduction) and surplus hemodynamic energy (SHE; $>97 \%$ reduction). ${ }^{2,3}$

Long-term CFVAD support and the resulting diminished pulsatility have been associated with clinical complications, including gastrointestinal bleeding, hemorrhagic strokes, arteriovenous malformations, and compromised end-organ function. ${ }^{1}$ However, the cellular-level adaptations and underlying mechanisms of clinical complications caused by diminished pulsatility have not been fully understood.

\footnotetext{
${ }^{1}$ These authors contributed equally to this work as co-first authors.

${ }^{2}$ These authors are both co-corresponding authors.
}

In this preliminary study, we used an endothelial cell culture model (ECCM) to evaluate the cell morphology and intracellular redox changes (i.e., gene and protein expressions for molecular redox signature) in response to diminished pulsatility in human endothelial cells. The ECCM accurately mimics normal hemodynamics and features of the vasculature, including cyclical pressure, shear stress, and axial and radial stretch. ${ }^{3}$

Human aortic endothelial cells (HAECs) cultured in the ECCM platform were subject to cyclic pressure, flow, and stretch conditions observed in normal individuals $(n=4)$ or I patients with CFVAD support ( $n=4-8$; Figure 1A). The pressure and flow waveforms were modulated accordingly to ensure that mean arterial pressure and mean flow rate for the normal and CFVAD conditions were matched and maintained at $95 \pm 1 \mathrm{~mm} \mathrm{Hg}$ and $9 \pm 0.5 \mathrm{ml} / \mathrm{min}$, respectively, whereas various other parameters, such as peak systolic pressure, diastolic pressure, mean arterial pressure, peak systolic flow rate, mean diastolic flow rate, energy-equivalent pressure, and SHE, were comparable to clinically observed values for both normal and CFVAD support (Figure 1B).

The HAECs were cultured on membranes with similar elastic modulus as the human aorta. The imposition of CFVAD pressure profiles resulted in a nearly constant stretch value of $\sim 7 \%$, whereas pressure associated with normal pulsatile flow resulted in cyclic stretch of between $6 \%$ and $10 \%$. The conditions experienced by HAECs within the ECCM system were subject to nearly physiologic values, with particular attention being paid to energyequivalent pressure and SHE values.

HAECs were evaluated after 96 hours in culture. For both normal and CFVAD support conditions, cells were maintained under normal pulsatile flow for 24 hours to ensure adaptation to flow conditions and then continued under normal flow or with CFVAD flow for the remaining 72 hours. After 96 hours, morphology, cytoskeletal organization, and mitochondrial distribution were analyzed in HAEC under CFVAD vs normal flow (Figure 2A).

Microscopic analysis revealed apparent morphologic changes in HAECs under CFVAD flow. Cells subjected to CFVAD appear as a circular phenotype with significant cytoskeletal filament disorientation to the periphery of the cell in relation to cells cultured under normal pulsatile flow, which have a naturally elongated phenotype and prominent distribution of cytoskeletal filaments. Morphologic changes associated with CFVAD flow also resulted in initiation of a proliferative response in HAECs, as observed from increased cell numbers and growth of cells in multiple layers. We subsequently stained the cells with MitoTracker (Molecular Probes, Life Technologies) to determine the changes in mitochondrial distribution and did not observe significant changes. 